\title{
Familie und Schule: zwei Orte der Erziehung
}

Hartmann Tyrell \& Raf Vanderstraeten

Widmet man der historischen Evolution der Erziehung Aufmerksamkeit, dann springt die schnelle Expansion der Schulerziehung seit etwa der Mitte des 18. Jahrhunderts ins Auge. In einem begrenzten Zeitraum wird die Schule zu einer selbstverständlichen Station im Erziehungsprozess für fast jeden Heranwachsenden. Der Erziehungsprozess ist dabei auf (wenigstens) zwei unterschiedliche Milieus verteilt: Erziehung findet in der Familie und in der Schule statt. Diese Innendifferenzierung des Erziehungssystems, die Differenzierung von Familie und Schule, prägt seitdem die Identitätsentwicklung jedes Heranwachsenden. In der zweiten Hälfte des 18. Jahrhunderts fanden hier grundlegende Änderungen statt, die in wenigen Jahrzehnten Tatsachen und Bewusstseinslagen verändert haben. In vielerlei Hinsichten sind Entscheidungen getroffen worden, die bis heute nachwirken. „Die Differenzierung von Familie und Schule - sowohl im Nacheinander der Biographie als im Nebeneinander des Lebensvollzugs - ist die erste Differenzierung von Differenzen, die erste Systemdifferenzierung, der der Heranwachsende sich ausgesetzt sieht, und es muss von unabschätzbarer Auswirkung sein, wenn jedermann heute auf diesem und keinem anderen Wege seine Identität suchen und binden muss“, so Niklas Luhmann und Karl Eberhard Schorr (1982: 246f.).

Die historische Transformation der Erziehung ist in einen ,katastrophalen' gesellschaftlichen Transformationsprozess eingebunden. Sie ist Bestandteil des Übergangs zu einer Gesellschaftsformation, die primär durch die Form der funktionalen Differenzierung gekennzeichnet ist. Die im 18. Jahrhundert anlaufende Ausdifferenzierung eines Erziehungssystems für die Gesamtbevölkerung entspricht zunächst den Anforderungslagen und Unterstützungsbereitschaften, die vor allem in den Bereichen von Politik und Wirtschaft gegeben waren. Häufig wird dabei betont, dass der Aufbau von nationalen Unterrichtssystemen Bestandteil eines umfassenderen Prozesses ist, wobei Familien und Haushalte ihre traditionalen Aufgaben oder Funktionen teilweise an funktional spezialisierte Organisationen abgeben (z.B. Inkeles/Sirowy 1983). Gleichzeitig kann aber behauptet werden, dass die Familie nur als Folge des Verlustes von bestimmten (wirtschaftlichen, politischen etc.) Funktionen ihre eigene(n) Funktion(en) hat finden können - und dass deswegen auch ihre gesellschaftliche Bedeutung zugenommen hat.

So meint Talcott Parsons (1955: 9f.), dass die moderne Gesellschaft von einer Struktur gekennzeichnet ist, in which the family is more specialized than before, but not in any general sense less important, because the society is dependent more exclusively on it for the performance of certain of its vital functions“. Parsons geht es dabei, psychoanalytisch inspiriert, vor allem um die Sozialisationsfunktion der Familie. In der modernen Gesellschaft wird die Familie zudem pädagogisch sensibilisiert. ${ }^{1}$ Im Erziehungsprozess nimmt die

1 Parsons zufolge übernimmt Sozialisation für das Sozialsystem Gesellschaft die Funktion, die laufende Reproduktion sozialer Beziehungen zu sichern. Erziehung kann dann als beabsichtigte Sozialisation definiert werden. Sie erfüllt dieselbe Funktion unter anspruchsvolleren Bedingungen, die eine systematische, lehr- und lernmäßige Intentionalisierung der Sozialisationsprozesse erfordern. Wenn man ,Sozialisation` als Grundbegriff wählt, kommt nicht zuletzt die Bedeutung der Familie für die Identitätsentwicklung der Heranwachsenden in den Blick. Nicht zufällig veröffentlichen Parsons und Bales ihre Reflexionen unter dem Titel Family, Socialization and the Interaction Process (1955). Man kann mit Claessens (1967) auch von der zweiten, sozio-kulturellen 
Bedeutung der Schule zu - jedoch nicht auf Kosten der Familie. Aber auch die Bedeutung der Familie wächst in diesem Prozess. Das legt die Idee eines Steigerungsverhältnisses nahe und bildet unseres Erachtens einen interessanten Ausgangspunkt für die Analyse der Innendifferenzierung des Erziehungssystems.

Die Ausdifferenzierung der Familie und ihre sozialisatorische Bedeutung für die Identitätsentwicklung der Heranwachsenden wurde z.T. schon früher von einem von uns analysiert (siehe Tyrell 1980, 1982, 1987). In diesem Beitrag wählen wir - im Anschluss an Arbeiten von Jürgen Markowitz (siehe vor allem Markowitz 1986) - den komplementären Ausgangspunkt, nämlich die Ausdifferenzierung der Schulerziehung. Aus dieser Perspektive soll hier das Verhältnis zwischen Familie und Schule in den Fokus rücken. Die Analyse ist in drei Punkte gegliedert. Zuerst soll näher auf einige Charakteristika der pädagogischen Semantik des 18 . Jahrhunderts eingegangen werden, die vor allem die Bedeutung der Familienerziehung betont. Anschließend wird die Verschiebung des Interesses erläutert: Schule und Schulerziehung rücken in der zweiten Hälfte des 18. Jahrhunderts nachdrücklicher in das Zentrum der Aufmerksamkeit. Zum Schluss werden unterschiedliche Dimensionen der Differenzierung von Familie und Schule erläutert und ihre Konsequenzen für das Heranwachsen von Kindern und Jugendlichen. Insgesamt hat dieser Beitrag nur explorierende Ambitionen. Uns geht es um die Schärfung der Beobachtungsmöglichkeiten; dagegen geht es uns nicht um das Aufzeigen kausaler Faktoren, die das Verhältnis zwischen Familie und Schule im Erziehungssystem bestimmen.

\section{I.}

Im Erziehungskontext ist die Schule häufig der Familie gegenübergestellt worden. Die These, dass Schulerziehung eine Bedrohung für die Familie darstellt, wird gewöhnlich mit mehreren Argumenten untermauert. So hat man gemeint, dass die Schule die ,natürlichen' elterlichen Verantwortlichkeiten untergräbt, weil sie sich Aufgaben aneignet, die ,eigentlich' den Eltern zustehen. Auch würde die Schule den Einfluss der Eltern und der elterlichen Werte und Normen zerstören, weil sie dem Einfluss des ,Schulmeisters' Vorschub leistet und weil dieser Einfluss anders ist und möglicherweise auch stark kontrastiert mit dem der Eltern. Außerdem wird die Schule häufig als eine potentielle Quelle moralischer Korruption betrachtet. Eltern haben wenig oder gar keine Kontrolle über die Mitschüler, mit denen ihre Kinder in der Schule Kontakt haben. Für die Eltern besteht die Gefahr, dass ihre Kinder schlechte Freundschaften schließen oder mit den falschen Leuten in Kontakt kommen. In ,besseren' Kreisen Englands etwa wurden die Kinder aus diesen Gründen auf exklusive Privatschulen geschickt und bekamen dann zugleich detaillierte Instruktionen hinsichtlich ihrer Mitschüler, mit wem und mit wem nicht verkehrt werden darf. Wenn schon die Moral der Kinder nicht gesichert werden

Geburt des Menschen in der Kernfamilie sprechen; zum Sozialisationskonzept und seiner Geschichte instruktiv Danziger (1974). Wenn man ,Erziehung‘ als Ausgangspunkt wählt, liegt es nahe, vor allem der Schule Aufmerksamkeit zu widmen. 
kann, so soll doch wenigstens versucht werden, den sprachlichen Akzent oder die Selbstdarstellungsweise der ,besseren' Kreise zu erhalten.

Häufig wurde die Schule als eine reelle Gefahr für die Familie angesehen - so real, dass einflussreiche soziale Reformer sich wiederholt gegen die Ausbreitung von Schulen und Unterrichtseinrichtungen ausgesprochen haben. Ihr Widerstand basierte auf der Vorstellung, dass die Schule die Familie nicht untergraben sollte. Leistungen, die Familienmitglieder, wie inadäquat auch immer, füreinander erbringen können, sollten nicht von bezahlten und ,seelenlosen Mietlingen ‘ erbracht werden. Diesem Argument der ,Heiligkeit der Familie‘ ist später ein psychologisches Argument hinzugefügt worden. Besonders in Bezug auf junge Kinder wurde und wird behauptet, dass Kindertagesstätten, Auffangheime und Schulen sowohl eine Gefährdung für die natürliche Beziehung zwischen den Eltern und ihren Kindern als auch für die Entwicklung der Kinder selbst darstellen. Seit den Publikationen von John Bowlby wird erheblicher Wert gelegt auf attachment: d.h. auf Sicherheit, Vertrauen und Wärme in der Beziehung des Erwachsenen zum Kind. Die Vorstellung ist, dass die unvermeidlichen Kontraste zwischen den Regelsystemen der unterschiedlichen sozialen Milieus, in denen Kinder heranwachsen, die psychische Entwicklung des Kindes bedrohen. „Manchmal haben Kindergärten die Ambition, sich zu profilieren. Diese Ambition kann ihren Ausdruck in dem Wunsch der Kindergarten-Leitung finden, einen eigenen pädagogischen Kurs zu verfolgen, eventuell gegen den der Eltern. Es ist offensichtlich, dass dies wohl das Letzte wäre, was ein normaler Kindergarten unter normalen Umständen machen sollte. Wir müssen unsere Hände nicht schmutziger machen wollen, als sie schon sind“, so äußerte sich noch vor kurzem der niederländische Pädagoge Jan Dirk Imelman (1998: 146, Übersetzung die Verf.).

In gewissem Sinne geht es hier um ein Echo von Auffassungen, die schon viel früher geäußert worden sind. Lange bevor man sich auf John Bowlby berufen konnte, wurde bereits gegen die Ausbreitung des familienexternen Unterrichtssystems protestiert. Schon in der ersten Hälfte des 19. Jahrhunderts mussten die Verfechter der vorschulischen Erziehung argumentieren, dass diese Einrichtungen, wenn sie landesweit verbreitet würden, die Nation nicht korrumpieren und die Eltern keineswegs von ihren Pflichten ,befreien' würden. Vor diesem Hintergrund formulierte Samuel Wilderspin, einer der bekannten Verfechter der Ausbreitung dieses Erziehungstyps in England, einige rhetorische Fragen in seinem The Infant System (1840, ch. 3): „Where are the natural guardians of the child? Where are its parents? Are we to encourage their neglect of duty, by becoming their substitutes?" Seine Antwort lautete wie folgt: Es geht nicht um den Gegensatz von Familie und Schule. Die Gefahr für die Entwicklung kommt von anderswo her. Sie kommt von den anderen Milieus, mit denen das Kind in Berührung kommen kann, vor allem von ,der Straße', von ihrer Kriminalität und den Drogen (Alkohol etc.). Der Mangel an Begeisterung für den Kindergarten zeigt sich auch in dem sehr zurückhaltenden Urteil von Sir James Kay-Shuttleworth, der als einer der großen Pioniere des englischen Schulsystems bekannt geworden ist. In einem historischen Überblick über die englischen Infant Schools, die für Kinder vom 2. oder 3. bis zum 6. Lebensjahr gedacht waren, wird der Erfolg dieser Schulen betont. Aber gleichzeitig wird der Hoffnung Ausdruck verliehen, dass diese Einrichtungen in der Zukunft nicht mehr vonnöten seien - nämlich wenn „parents more lettered and less sensual, will be less prone to neglect infants and children of a riper age“" (Kay-Shuttleworth 1862/1993: 132). 
Im 18. und 19. Jahrhundert blieb die Debatte in England nicht beschränkt auf das Niveau der vorschulischen Erziehung. Sie bezog sich auf das Unterrichtssystem im Ganzen, auf die Existenzberechtigung eines landesweiten Netzwerkes von Schulen und Erziehungseinrichtungen. Es wurde vorausgesetzt, dass die Schule nicht notwendig war für Kinder, die in ordentlichen und anständigen, gut funktionierenden Familien aufwuchsen, die all dasjenige organisieren könnten, was von ordentlichen Familien zu erwarten sei (vom Brotbacken und Organisieren des Unterrichts bis hin zum Durchführen kleinerer chirurgischer Eingriffe einschließlich von Abtreibungen). Nur wo Familien schlecht funktionierten, sei es notwendig, die Kinder den moralischen Gefahren und der Barbarei des Schullebens auszusetzen. Wenigstens bis zum Ende des 19. Jahrhunderts fand die Idee Beifall, dass der Schulbesuch der Kinder die Familie selbst in Misskredit bringen könnte. Ein Schulbesuch deutete darauf hin, dass die Eltern und die nächsten Familienmitglieder ihren Aufgaben nicht gewachsen waren und diese anderen Leuten überließen. ,Gute Eltern“ waren nicht die, die ihr Kind zur Schule schickten, sondern die, die dies gerade nicht taten (vgl. Smelser 1990). ${ }^{2}$

Eltern wurde davon abgeraten, ihre Kinder zur Schule zu schicken. Schuleinrichtungen, die Kinder aktiv zu rekrutieren versuchten (z.B. mit bestimmten finanziellen oder materiellen Vorteilen), wurden offen kritisiert - auch von Philanthropen und sozialen Reformern. Die Eltern, meinte man, waren am besten im Stande ,to train up their children as olive plants round the domestic table, and rear them within the tender, kind, holy and heaven-blessed circle of a domestic home. There are nursed those precious affections towards parents, brothers, sisters and smiling babes, which, for man's good in this life, and the wellbeing of society, are worth more than all the Greek and Roman lore" (Guthrie 1860/1973: 3). Auf Grund der pädagogischen Idealvorstellungen dieser Zeit darf behauptet werden, dass Schulen im Grunde ein Symptom des Versagens der Familie waren. Ihre Existenzberechtigung verdankten sie nur der Tatsache, dass bestimmte Eltern ihren pädagogischen Aufgaben nicht gewachsen waren und/oder bestimmte Kinder sich als besonders widerspenstig und ungehorsam erwiesen. Die Eltern wurden ermahnt, ihre Kinder im Hause zu erziehen und unterrichten. Die Schule galt als ,,a general infirmary for mental disease, to which all desperate subjects are sent, as the last resource", wie die Edgeworths in einem Buch mit dem Titel Practical Education (1801/1992: 503) behaupteten; dies Buch ist noch immer bekannt als ,the most outstanding treatise on education in circulation during the first half of the nineteenth century" (Curtis/Boultwood 1962: 111).

Im Sog von Jean-Jacques Rousseau, dessen Emile 1762 veröffentlicht wurde, sind eine ganze Reihe pädagogischer Traktate und Abhandlungen publiziert worden. Dabei auch eine Reihe von Publikationen (teils theoretischer, teils praktischer Art), die sich auf die Erziehung der Kinder durch ihre eigenen Eltern konzentrierten. Vor allem wurde betont, dass die natür-

2 Siehe zum Beispiel die autobiographischen Skizzen seiner Kindheit, die Hugh Millar unter dem Titel My Schools and Schoolmasters publiziert hat. Mit seiner Erziehung waren seine Onkel betraut, weswegen er gemeinsam mit seinen Cousins erzogen wurde. Religionsunterricht wurde durch diese Onkel selbst erteilt, obwohl auch Sonntagsschulen diese Aufgabe übernahmen. „But Sabbath schools my uncles regarded as merely compensatory institutions, highly creditable to the teachers, but very discreditable indeed to the parents and the relatives of the taught; and so they of course never thought of sending us there" (1865: 37). Ganz ähnliche Gedanken über Erziehung hatte John Locke (1693/1878) bereits am Ende des 17. Jahrhunderts formuliert. 
liche Erziehung mehr Beachtung verdient - und dann nicht allein die Erziehung in einer idyllischen Natur (wie bei Rousseau), sondern auch die Erziehung durch die natürlichen Erzieher: die Eltern (Rousseaus Emile war ein Waisenkind). In verschiedenen Publikationen wurde gleichzeitig Stellung gegen die Zivilisation und die Kultur genommen, wobei die Schule und die schulischen Unterrichtsmethoden als Kultursymbol par excellence dargestellt wurden. So hielt unter anderem David Williams in seinem Treatise on Education Ausschau nach einer Zeit, in der Menschen ,[would be] capable of presiding over their own families and educating their children; and render schools themselves unnecessary“ (1774: 103). Im gleichen Jahr beschrieb Willem Emery de Perponcher dieses Ideal in seinen Instructions d'un père à un fils (hier trug der Sohn übrigens den viel sagenden Name Emile). Einige Jahre später hat er noch einmal zur Feder gegriffen und eine mehrteilige, stärker an der Erziehungspraxis orientierte Arbeit über Privaterziehung geschrieben, Onderwijs voor Kinderen (1782 [d.h. Unterricht für Kinder; die Verf.]), die in den Niederlanden lang einflussreich blieb. ${ }^{3}$

Trotz dieser deutlichen Präferenz für die Familie kamen auch Mischformen von Familienerziehung und Schulerziehung vor. Manche Autoren befürworteten Systeme privaten Unterrichts im Hause, zu denen man auch andere Jugendliche hinzuzog, um auf diese Art und Weise soziale Erfahrungen, Nachahmung und Wettbewerb zu ermöglichen. Davon sind in der Literatur schon früh Beispiele zu finden (z.B. Mulcaster 1581). In der zweiten Hälfte des 18. Jahrhunderts tauchen solche Stimmen häufiger auf. „A number of gentlemen of fortune, whose sons are nearly of an equal age, and free from all tincture of vice, and are all provided with separate tutors [should] contrive to bring them often together, so as to perform certain exercises in common", so lautete zum Beispiel die Empfehlung von Joseph Priestley (1788: 75). Hier wird deutlich, dass der Adel sich das Risiko vergegenwärtigte, mittels der Privaterziehung seinen Selbstausschluss aus der Gesellschaft zu organisieren. Zudem wurde man sich im Übergang zur modernen Gesellschaft deutlich bewusst, dass es neben dem Stand auch einen Beruf gab und dass man diesen wählte und nicht unbedingt vom Vater übernahm. Väter, die selbst durch ihre Berufstätigkeiten völlig in Anspruch genommen waren, verfügten auch über zu wenig Zeit für die Erziehung ihrer Söhne. Vor diesem Hintergrund erschienen dann Familie und Schule, private und öffentliche Erziehung, allmählich weniger als disjunktive Alternativen, als gegensätzliche und einander bedrohende Welten (vgl. Stichweh 1991; Vanderstraeten 2004).

3 In den Jahren nach der Publikation des Emile haben verschiedene Eltern auch versucht, ihre Kinder tatsächlich nach Rousseaus Prinzipien zu erziehen. Über die Erfolge bleiben die Meinungen geteilt (siehe dazu z.B. Douthwaite 2002; Baggerman/Dekker 2005). Allerdings: die Kindheit Herbert Spencers war auch durch eine solche experimentelle Vorgehensweise gekennzeichnet (siehe Spencer 1904). Was die Rezeption Rousseaus in Deutschland betrifft, darf die Bedeutung der philanthropischen Pädagogik nicht unerwähnt bleiben. Eine reichlich kommentierte Übersetzung des Emile erschien in den letzten Bänden der Encyclopedia Allgemeine Revision des gesamten Schul- und Erziehungswesens. Für die pädagogische Praxis waren die Publikationen und Schulgründungen der Philanthropen vermutlich bedeutsamer als die philosophischen Abhandlungen Rousseaus. 
II.

Das Europa des späten Mittelalters und der frühen Neuzeit lässt sich als eine ständisch gegliederte Gesellschaft verstehen. Sie war primär differenziert in Subsysteme aus hierarchisch angeordneten, voneinander getrennten ständischen Gruppen, denen die Individuen und Haushalte jeweils mit der Gesamtheit ihrer Lebensführung zugehörten. Die Rangordnung bestimmte die Verhältnisse untereinander bzw. die relevanten Unterschiede. Dabei wurden Familie und Haushalt als die natürliche Umwelt eines jeden Individuums betrachtet. Daran wird auch im 19. Jahrhundert noch wiederholt appelliert. „God never made men to be reared in flocks, but in families. Man is not a gregarious animal, other than that he herds together with his race in towns, a congeries of families. Born, as he is, with domestic affections, whatever interferes with their free play is an evil to be shunned, and, in its moral and physical results, to be dreaded. God framed and fitted man to grow up (...) [under] the domestic roof" (Guthrie 1860/1973: 2f.). Im Idealfall war die Familie eine selbständige, autarke Einheit, worin auch Erziehung enthalten sein sollte. Das Haus selbst schien also auf den ersten Blick der ideale Kontext, um alles, was für seine Erhaltung wichtig war, zu vermitteln. Alles, was für den Fortbestand oder die Fortführung des eigenen Hauses wichtig war, sollte am besten innerhalb der und durch die Familie vermittelt werden. Gleichzeitig schienen Familienstruktur und Gesellschaftsstruktur gut aufeinander abgestimmt. Die hierarchisch strukturierte Mikrowelt der Familie konnte die Kinder dann auch bestmöglich auf die makrosozialen Herrschaftsverhältnisse vorbereiten.

Diese Auffassungen wurden aber als Folge von einer Reihe weit reichender gesellschaftlicher Transformationen in Frage gestellt. Im 18. Jahrhundert artikulieren sich andere, neue Erwartungen in Bezug auf Erziehung und Unterricht. In Europa hat die Expansion der Schule und des Schulunterrichts in der zweiten Hälfte des 18. Jahrhunderts einen Anfang genommen. Dieser Zeitraum brachte ebenfalls ein geradezu explosives Wachstum pädagogischer Publikationen (häufig als Reaktion auf die Publikation von Rousseaus Emile aus dem Jahre 1762). In den Niederlanden z.B. erschienen in den 60er Jahren des 18 . Jahrhunderts mehr pädagogische Abhandlungen als in den gesamten vorangegangenen 100 Jahren. Diese bemerkenswerte quantitative Zunahme der Publikationstätigkeit ging mit einer Verschiebung der Ausgangspunkte der Debatten über (den Ort der) Erziehung einher. Auch als Folge der schnellen Ausbreitung der Volksschule kamen die herkömmlichen pädagogischen und gesellschaftlichen Idealvorstellungen immer stärker unter Druck.

Das geht unter anderem aus der Kritik über die Art und Weise hervor, wie Eltern ihre Erziehungsaufgaben erfüllen. Wer das Ideal der Privaterziehung im Elternhaus befürwortet, hat dann häufig kaum Vertrauen in die tatsächlichen Kompetenzen der Eltern. Die Schule wird dann manchmal als ,second best' Wahl präsentiert, als eine Option, die sinnvoll ist, eben weil die Eltern ihre Erziehungspflichten vernachlässigen. „If all mothers fulfilled their duties, there would be little occasion for boarding schools; but if they give up their time and attention to dress, to visiting, to cards, to public places, it is better that the children should go to school, than that they should converse with servants, or play in the streets", so zum Beispiel Clara Reeve (1792/1974: 73). Ein breit formuliertes Preisausschreiben eines niederländischen Vereins (Provinciaal Utrechtsch Genootschap van Kunsten en Wetenschappen) sondierte 1793 
auch die Grenzen zwischen beiden Formen der Erziehung: Erziehung im Elternhaus oder in der Schule. „Welche Art der Erziehung ist am meisten zu favorisieren? Eine öffentliche oder eine private? Welche sind die Vorteile und Mängel der beiden? Gibt es eine Art der Erziehung, die zwar die Vorteile von beiden, nicht aber deren Nachteile enthält?" In einer der preisgekrönten Einsendungen formulierte G.C.C. Vatebender seine Position wie folgt: „Die Erziehung im Hause wird, entweder von den Eltern selbst oder unter ihren Augen durchgeführt; häufig jedoch auch völlig ohne die Aufsicht der Eltern und ohne die Aufmerksamkeit der Eltern zu erhalten, durch Hausmädchen oder Aufseher; oder sie wird von Hauslehrern und Hauslehrerinnen erteilt, die selbst noch der Erziehung bedürfen“" (1793/1801: 3, Übersetzung die Verf.). Schulerziehung wird verteidigt mittels einer Kritik der Mängel und Beschränkungen der Privaterziehung. Die anderen preisgekrönten und publizierten Einsendungen verfechten in dieser Hinsicht keine anderen Positionen.

Die Beobachtung der pädagogischen Inkompetenz der Eltern ist das Pendant zu den wachsenden gesellschaftlichen Erwartungen in Hinsicht auf Erziehung und Unterricht. Politische und wirtschaftliche Entwicklungen unterstützten dabei die Realisierung eines landesweiten Netzwerks von Schuleinrichtungen. 1796 schrieb zum Beispiel August Hermann Niemeyer in Grundsätze der Erziehung und des Unterrichts für Eltern, Hauslehrer und Erzieher: „Seit man häusliche und öffentliche Erziehung unterschieden und beyde Arten versucht hat, ist auch darüber gestritten, ob die eine oder die andre die bessere sey? Es fehlt von beyden Seiten nicht an Gründen“ (1796/1970: 15). Die pädagogische Autorität und die Arbeitsfreude des Lehrers sind zum Beispiel Themen, die bereits im 18. Jahrhundert die Debatten über die Vor- und Nachteile der Schulerziehung im Vergleich zur Familienerziehung stark prägten. So wurden pädagogische Autoritäts- und Vertrauensprobleme im Hinblick auf die größere Auffälligkeit und Unverzeihlichkeit von Fehlern der Lehrer im Vergleich zu solchen der Eltern diskutiert (siehe Ehlers 1766: 165ff., 213ff.). Um die Jahrhundertwende wird die Schule aber so sehr Zentrum aller Erziehungs- und Reformüberlegungen, dass die Pädagogik sich mehr und mehr auf den Schulunterricht konzentriert. Dann wird auch behauptet und vorausgesetzt, dass der Pädagoge oder Schullehrer, dem die, natürlichen “ Autoritätsgrundlagen der Eltern fehlen, einen Ersatz in seiner professionellen Kompetenz finden kann (siehe z.B. Trapp 1780/1977).

Der Widerstand gegen Schulerziehung darf übrigens nicht unterschätzt werden - besonders nicht in Ländern, wo ein Standesbewusstsein stark kultiviert wurde und wird. Besonders in England waren im 19. Jahrhundert die ,happy few', die sich auf die Tradition des eigenen Haushalts beriefen, noch zahlreich. Ein nicht unbeträchtlicher Teil der Kinder aus den ,besseren' Kreisen wurde auch im 19. Jahrhundert noch im elterlichen Haushalt auf die Universität oder das Berufsleben vorbereitet. Laut Statistiken, die von offiziellen englischen Kommissionen publiziert wurden, hatten 1868 noch 11,6\% der Studierenden, die an den Universitäten von Oxford und Cambridge immatrikuliert waren, vorab nur eine Privaterziehung im Haushalt der Eltern genossen. 30 Jahre später waren noch immer 11,4\% der Bevölkerung der weiterführenden Schulen zuvor nur im Hause der Eltern unterrichtet worden (siehe Hans 1951; Musgrove 1966: 16ff.; Smelser 1991: 39ff.). Für die englischen , happy few' haben die so genannten ,public schools“ (Eton, Winchester etc.) später die Familie als primäres Erziehungsmilieu ersetzt; die Empfindsamkeit für Rangunterschiede hat sich auf diese Art und Weise aber keineswegs verringert. 
Die Einführung der Schulpflicht ist ein typisches Phänomen des 19. Jahrhunderts. Die diesbezügliche Gesetzgebung ,[is] adopted in virtually every Western European country in the ,long' nineteenth century, from Prussia (1763) to Belgium (1914)“ (Ramirez/Boli 1987: 3). Durch diese Gesetzgebung wird die Teilnahme an Schulerziehung universalisiert. ${ }^{4}$ Im Prinzip bekam dann auch jeder auf die gleiche Weise Zugang zu Erziehung und Unterricht. Vor diesem Hintergrund kann die Einführung der Schulpflicht gesehen werden als eine Spezifizierung der Inklusionspostulate, wie sie unter anderem durch die Ideale der Französischen Revolution symbolisiert wurden (Freiheit, Gleichheit, Brüderlichkeit). Durch die allgemeine Schulpflicht wurde die Inklusion der Gesamtbevölkerung im Erziehungssystem garantiert (Luhmann/Schorr 1988: 29ff.). Für die Durchschlagskraft von universalen Inklusionspostulaten in der modernen, funktional differenzierten Gesellschaft scheint die Schule in der Tat ein klassischer Fall zu sein. Aber das impliziert nicht, dass Rangunterschiede außer Kraft gesetzt werden. ${ }^{5}$ Die Inklusionspostulate der modernen Gesellschaft sind nicht mit Selbstverwirklichungsmöglichkeiten ausgestattet (vgl. Vanderstraeten 2006).

Niklas Luhmann und Karl Eberhard Schorr haben, bezogen auf das Erziehungssystem, die Inklusionsthematik mit der so genannten Entdeckung des Kindes verknüpft. ,Nicht zuletzt wird durch das Festmachen am Kinde die universelle Inklusion der Gesamtbevölkerung in den Erziehungsprozeß begründbar; denn schließlich kommen alle hilflos auf die Welt, wachsen alle als Kinder auf, werden alle irgendwie durch ihre Umwelt erzogen, und es fragt sich nur: wie gut“ (Luhmann/Schorr 1988: 33). Die Entdeckung des Kindes unterstützte sicherlich die Interessen der Pädagogen (vgl. Ariès 1960; Heywood 2001; Wahrman 2004). Begriffe wie „Natur“, „Neigung“, „,Talent“ und „Kraft" tauchen erstmalig in diesem Zusammenhang auf und bezeichnen eine individuelle Disposition, die zumindest in Einzelfällen die soziale Hierarchie der Stände zu neutralisieren im Stande war (vgl. la Vopa 1988; Stichweh 1991). Dieser Idee nach entfallen alle besonderen Einschränkungen der Erziehung, die nicht ihr selbst, sondern ihrer ständischen Umwelt zuzurechnen sind. Anders formuliert: Soziale Systeme verbinden sich auf zirkuläre Weise mit ihrer sozialen Umwelt. Bei Karl Weick (1979) ist „enactment“ der Begriff, der den Prozess beschreibt, in dem Systeme eine Umwelt schaffen, die für sie dann später die letztlich orientierungsrelevante Umwelt bildet. Die neuen Konstrukte erlegen der Realität eine pädagogische Perspektive auf, die dann pädagogische Interventionen erfordert.

Die Neueinschätzung des Kindes ging einher mit einer ,redescription' der komplementären Leistungsrollen. Wir haben bereits darauf hingewiesen, dass die Erwartungen in Bezug auf die

4 Zur Ergänzung: In den meisten Ländern Europas gibt es keine Schulpflicht, sondern eine Lernpflicht. Das bedeutet, dass die Möglichkeit erhalten bleibt, dass Eltern ihre Kinder im eigenen Haushalt erziehen. Auch dabei können dann wieder alle wesentlichen Aspekte des Erziehungsgeschehens durch die Eltern selbst betreut werden, oder es werden Hauslehrer eingestellt, die speziell mit Erziehungsaufgaben befasst sind. Zur Zeit beobachtet man vor allem in den USA ein erneutes Interesse an ,home schooling' (für einen Überblick Bauman 2001).

5 Ein häufig erwähntes Thema am Ende des 18. Jahrhunderts. So meinte zum Beispiel Peter Villaume, dass der Erzieher, ,seinen Zögling nicht vollkommener machen [muss], als es sein Stand erlaubt; außer, wenn er sieht, daß dessen Kräfte ihn offenbar zu einem andern Stande bestimmen“ (1785: 526; ganz ähnlich: Wolff 1780/1977: 107f.). Auch für de Condorcet blieb jemandes Erziehung durch seinen Standort in der ständischen Hierarchie bestimmt: „Les enfants, suivant la richesse de leurs parents, les circonstances où se trouvent leurs familles, 1'état auquel on les destine, peuvent donner plus ou moins de temps à l'instruction“" (1792/1847: 188f.). 
Eltern (jetzt vor allem: die Mütter) zunahmen. Im 18. Jahrhundert fand eine pädagogische Sensibilisierung der natürlichen Erzieher statt (siehe Gilgenmann 1994). Gleichzeitig wurde auch die Frage nach ausgebildeten professionellen Praktikern (Schullehrern) in die Wege geleitet. „Was man mit Sorgfalt treiben muß, dazu muß man sich gehörig vorbereitet haben; man muß der Grundsätze desselben kundig sehn und ihre Anwendung sowohl theoretisch als praktisch erlernt haben. Das heißt mit andern Worten: Die Erziehung muß als eine eigne Kunst von ihren eignen Leuten getrieben werden“, so behauptete Ernst Christian Trapp (1780/1977: 21). Das Nichtausreichen der natürlichen Erziehung begründet den gesellschaftlichen Bedarf für Erziehung, auf den der Pädagoge sich berufen kann, wenn er Forderungen stellt oder sich zu rechtfertigen hat. Es begründet die Professionalität der Praxis des Lehrens und die Notwendigkeit einer spezialisierten Berufsvorbereitung. Trapp selbst wurde 1779 als allererster Professor für Pädagogik (in der Welt!) berufen - die Berufung fand selbstverständlich in Halle statt.

Vor allem seit der Mitte des 19. Jahrhunderts hat eine Aufwertung des Lehrerberufs stattgefunden. Der Beruf wird häufiger als vollwertig und nicht nur als eine Nebenbeschäftigung, die mit anderen Beschäftigungen (Bauer, Küster etc.) kombiniert werden kann, angesehen. Auch andere, zusätzliche Rollen können in diesem Sog stabilere Formen gewinnen. Schulrecht, Schulverwaltung, Schulaufsicht werden ausgebaut. Lehrplänen und Curricula wird mehr Aufmerksamkeit gewidmet. Im Laufe des 19. Jahrhundert entstanden auf die Art umfangreiche, organisierte Netzwerke von Schulen und Erziehungseinrichtungen; die Existenz dieser Netzwerke ist seitdem zu einer Selbstverständlichkeit geworden. Und erst nachdem die ausdifferenzierte Schulerziehung als Fokus aller Fortschritte ins Zentrum der pädagogischen Aufmerksamkeit rückt, kann die Familienerziehung mit Bezug auf Schule neu durchdacht werden. Mit der Inklusion der Gesamtbevölkerung in die Schule ist das (biographisch-temporäre) Doppelleben von Schulkindern in Familie und Schule jedenfalls gesellschaftliches Gemeingut geworden.

Die Gegenüberstellung beider Milieus bleibt aber erhalten. So scheint sowohl die Familie von der Schule her als auch die Schule von der Familie her in spezifisch pädagogischer Hinsicht noch immer strukturell diskreditierungsanfällig. Das Gefälle an professioneller Kompetenz zwischen Lehrern und Eltern ist immer zu Lasten der letzteren auslegbar. Diese sind pädagogische Laien und selbst bildungsbedürftig; das entsprechende Programm heißt Elternbildung. Oder auch: Vor den schulischen Ansprüchen gerät die familiale Sozialisationsleistung leicht in die Situation des Defizitären und Kompensationsbedürftigen. Umgekehrt sind von der Familie her immer Organisationsressentiments gegen die Schule mobilisierbar. Diese ist dann leicht diskreditierbar als kalter, liebloser, ja inhumaner Ort, wo ein Haufen Kinder von Unterrichtsbeamten allenthalben verglichen, gemessen, zensiert und selektiert wird. Auch Schüler sind dort nicht immer glücklich: „Unterricht bleibt relativ ereignisarm; Schüler registrieren es bereits mit Dankbarkeit, wenn versehentlich einer ihrer Kameraden mit dem Stuhl umkippt“" (Markowitz 1982: 92f.). Deutlich ist: Die Pädagogik kann dem strukturellen Dualismus von Familie und Schule wenig Positives abgewinnen. Für das Wie der Aufgabenteilung zwischen den beiden getrennten Erziehungsmilieus und beider Verhältnis zueinander fehlt es weitgehend an konsolidierter Reflexion. Die Pädagogik versagt hier als, Systembetreu- 
ungswissenschaft'. Aber auch im Bereich der Soziologie ist die Differenzierung von Familie und Schule bisher kaum näher analysiert worden.

\section{III.}

Die Differenzierung und Trennung von Familie und Schule steht in unverkennbarer Parallelität zu einem anderen Differenzierungsresultat, das für die moderne Gesellschaft in besonderer Weise kennzeichnend geworden ist: der Trennung nämlich von Haushalt und Betrieb, von Familien- und Berufsleben. In der Literatur wird diese Analogie für das Verhältnis von Familie und Schule geltend gemacht; dabei ist auch nachdrücklich von der Trennung von Lebens- und Erziehungsraum als Kennzeichen der Moderne gesprochen (siehe z.B. Fend 1980: 115ff., 2006). Dem ist weitgehend zu folgen. Allerdings gilt es auch, die Grenzen dieser Analogie zu sehen, wobei deren Benennung sogleich auf die Problematik der Interaktion von Elternhaus und Schule führt. Die vertrags- und geldvermittelte „Trennung von Berufs- und Familienrolle“ (Parsons 1972: 100) ist bezüglich des Trenneffekts schärfer geschnitten als die von Schul- und Familienrollen: Schulen können den Familienhintergrund ihrer Schüler, also primär deren Eltern, nicht in dem Maße neutralisieren und ignorieren, wie dies Betriebe hinsichtlich ihres Personals tun. Sie müssen die Eltern als eine legitime Bezugsgruppe in ihrer Umwelt, auf welche Art auch immer, akzeptieren: Lehrer können sich schwerlich verweigern, wo Eltern , advokatorisch' für ihr Kind in der Schule vorstellig werden; Elternsprechtage und Elternabende sind als schulische Interaktionsofferten institutionalisiert (siehe Maclure/Walker 2000); mitunter auch halten sich Lehrer ihrerseits, wo sie mit Schülern besondere Probleme haben, direkt an deren Eltern und setzen, wo sie selbst nichts ausrichten, auf elterliche Kontrolle. $\mathrm{Zu}$ alledem gibt es im Verhältnis von Betrieb und Familie nichts Paralleles; Grenzziehung und Trennung zwischen System und Umwelt wirken hier noch erheblich stärker , distanzierend‘.

In ganz ähnlicher Weise hat Talcott Parsons auf die Eigendynamik von Familie und Schule als differenzierte gesellschaftliche Teilsysteme hingewiesen. Bei Analysen der amerikanischen Normalfamilie schreibt Parsons (1955: 33): „But looked at as a part of the society, the family is, even in primitive societies, a specialized, i.e., differentiated, part of a larger system; it is quite erroneous to regard it as a ,microcosm" of the whole“. In Parsons" Analyse der amerikanischen Universität heißt es: „Although socialization in higher educational institutions helps prepare for citizenship roles in a societal community which emphasizes institutionalized individualism, the academic community is not a microcosm of the society but a differentiated part of the society" (Parsons/Platt 1973: 203). An diesen Ausgangspunkten wollen wir festhalten (vgl. Dreeben 1968). Die an- und abschließenden Überlegungen wollen vor diesem Hintergrund die Trennung von Familie und Schule genauer in den Blick nehmen. Die Absicht ist dabei, in relativ deskriptiver Manier aufzuklären, was diese Trennung strukturell besagt und wie diese Trennung sich im Erfahrungszusammenhang von Schulkindern faktisch darstellt. Dies soll stichwortartig, und ohne jeden Anspruch auf Vollständigkeit, in fünf Punkten $(\mathrm{a}-\mathrm{e})$ geschehen.

a) Die Differenzierung von Familie und Schule ist augenfällig zuerst eine räumliche: Familienleben und schulischer Unterricht finden an verschiedenen Orten statt. Oder auch von 
der Familie her (und analog zur Berufsarbeit): Das schulische Geschehen findet außer Hauses statt. ,In der Schule zu sein“ heißt folglich für das Kind: nicht ,zu Hause zu sein`. Hierbei ist vorausgesetzt, dass Familienleben und schulischer Unterricht jeweils an bestimmter (je anderer) Stelle lokalisiert sind: Hier wohnt das Kind, und dort geht es zur Schule. Eine Konsequenz ist, dass das Schulkind einen täglich zurückzulegenden Schul- bzw. Heimweg hat und sich dabei in einem Zwischenraum bewegt. Eltern und Lehrer tun sich häufig schwer damit, diesen Zwischenraum zu kontrollieren. Schüler andererseits nutzen gern die Möglichkeiten dieses Zwischenraums: Man raucht dort die erste Zigarette, man trifft dort den ersten Freund oder die erste Freundin oder die anderen Freunde etc.

Die räumliche und architektonische Struktur der Klassenzimmer setzt Lehrer zudem fast unvermeidlich einer intensiven Beobachtung durch ihre Schüler aus. Jedem einzelnen Schüler kann die ungeteilte Aufmerksamkeit des Lehrers nur in Ausnahmefällen gewährt werden, aber jeder Schüler soll kontinuierlich aufmerksam sein und hat dadurch hinreichend Gelegenheit, dem Lehrer bei der Beschäftigung mit der Schulklasse oder anderen Schülern zuzusehen. So sehr Lehrer auch versuchen, auf Distanz zu gehen, sie können es demnach nicht vermeiden, dem prüfenden Blick ihrer Klienten ausgesetzt zu bleiben. Sie werden, wie man leicht feststellen kann, schonungslos als normale, als all-zu-normale Personen beobachtet. Ihre Schwächen und Idiosynkrasien werden beobachtet und ausgenutzt und sind bei den Schülern ein permanent dankbares Gesprächsthema.

b) Des Weiteren ist die Differenzierung von Familie und Schule eine zeitliche. Das Kind hat täglich zu bestimmten Zeiten Schule - überdies nach festen Stundenplänen. Unbedingt gilt hier die Analogie zur täglichen Arbeitszeit im Beruf. Schule hat auch einen deutlich zeitlichen Sinn, der gerade auf zeitliche Begrenzung und mithin zeitliche Systemtrennung abstellt. Schule bzw. Unterricht ist etwas, das täglich zeitlich punktuell beginnt und auch endet. Wenn die Schule aus ist, stellen Kinder ihr Verhalten von einer Minute zur anderen drastisch um: Das Vorher und Nachher des zeitlichen Schulschlusses/Unterrichtsendes ist, ohne dass man das Schellen gehört haben muss, am Schülerverhalten unmittelbar ablesbar. Es bedarf kaum der Erwähnung, wie künstlich oder unwahrscheinlich eine solche Zeitorganisation mit ihrer punktuellen Systemgrenzziehung nach der Uhr ist und gerade für Kinder sein muss. Umso erstaunlicher ist es aber zu sehen, wie (relativ) problemlos die Kinder, die eine Uhr tragen, sich das hier gebotene Zeitverhalten aneignen, es als Normverhalten habitualisieren, auch wenn aus Lehrersicht einige es ,einfach nicht lernen wollen', pünktlich zu sein.

Familien ihrerseits haben sich intern in nicht minder erstaunlichem Maße auf die schulische Zeitorganisation, insbesondere die ihnen von dort, also extern vorgegebenen Zeitgrenzen, eingestellt. Die der Schule angepasste familiale Alltagsorganisation sieht - von den Ferien abgesehen - vor, dass das Kind tagtäglich rechtzeitig und angemessen gerüstet zur Schule geschickt wird, und das traditionelle deutsche Halbtagsschulsystem ließ (und lässt teilweise noch) synchron Millionen von Hausfrauen und Müttern Sorge tragen, dass die Kinder nach der Schule zu Hause erst einmal ihr Mittagessen einnehmen können. Andererseits können Schulferien Familien zu komplizierten Zwischenlösungen zwingen, wobei dann Großeltern, Freunde, Nachbarn etc. eingesetzt werden.

c) Die Trennung von Familie und Schule verlangt von Kindern weiterhin ein deutliches Auseinanderhalten der Systemreferenzen: Einerseits muss das Schulkind sich die Sinndifferenz 
zwischen Verhaltensweisen, die in die Familie gehören, die zu Hause angemessen sind, und solchen, die in der Schule (gegenüber den Lehrern und Mitschülern) richtig oder geboten sind, aneignen. Andererseits muss das Kind Verhaltenssicherheiten dergestalt aufbauen, dass es das richtige Verhalten am rechten Ort und zur rechten Zeit zur Verfügung hat, und auch, dass ihm nicht Verwechslungen unterlaufen (im Unterricht spielen wollen oder nach seiner Mutter rufen; zu Hause die Hand aufzeigen oder sich melden, wenn man etwas sagen will), mit denen es sich lächerlich macht. Um der Differenzierung von Schule und Familie gemäß adäquat agieren zu können, bedarf es der Einübung und stabilen Unterscheidung heterogener Verhaltenslinien, an denen dann auch Dritte auf Anhieb ablesen können, um welche Systemreferenz (Familie oder Schule) es sich handelt. ${ }^{6}$

Im Hinblick auf die für das Kind so massiv erlebbare Differenz von Familie und Schule ist wesentlich, dass die beiden heterogenen Verhaltenskomplexe unvermischt auf die beiden verschiedenen sozialen Orte verteilt sind und dass sie dort jeweils raum-zeitlich konzentriert betrieben werden: Die Schule ist nur oder doch primär der Ort des Unterrichts, der LehrerSchüler-Interaktion; das Familienleben, aber auch die Berufstätigkeit der Eltern gehören anderswohin, und entsprechend hat, getrennt davon, das (allerdings von den systemfremden ,Schularbeiten' durchsetzte) Familiengeschehen seinen häuslich-privaten Ort wesentlich für sich; anderes, etwa Schulunterricht, hat dort nicht seinen Platz. An der je unterschiedlichen Verortung sind die Systemreferenzen des Handelns anschaulich identifizierbar und im Hinblick auf Grenzbewusstsein differenzierbar.

d) Für die Kinder schlägt sich die institutionelle Trennung von Familie und Schule auch darin nieder, dass sie , allein ', ohne die Eltern, in die Schule gehen und dass sie sich nach der Schule ohne Lehrer und Mitschüler wieder auf ihre Familien verteilen. Dass sie normalerweise in der Schule ,ohne Eltern' und abgelöst von ihrer Familienumwelt agieren müssen, heißt vor allem: In der Schule sind die Eltern abwesend, und die Kinder befinden sich nicht in Interaktionsnähe zu ihnen. Schulkinder interagieren in der Schule mit Lehrern und Klassenkameraden ohne den Schutz, ohne die Rückendeckung anwesender Eltern; sie müssen hier, was zumal zu Beginn der Schulkarriere sehr schmerzhaft sein mag, allein zurechtkommen. Aber die Abwesenheit der Eltern bedeutet auch das Entfallen elterlicher Aufsicht und Kontrolle, sie hat sozusagen auch eine Freiheitsdimension. Wichtig ist ferner: Die nur ausnahmsweise Präsenz der Eltern in der Schule (erst recht im Unterricht) setzt den Lehrer normalerweise frei von der Notwendigkeit beständiger Mitberücksichtigung der Familienumwelt der Kinder.

Daraus ergibt sich, dass die Trennung von Familie und Schule eine Informationsseite hat, gerade weil davon auszugehen ist, dass Eltern im tagtäglichen Normalfall der Schule ihrer Kinder fernbleiben und von daher das Schulgeschehen für sie nicht gläsern oder gänzlich transparent ist. Beide Seiten weisen im Hinblick aufeinander ein strukturelles Informationsdefizit

6 Schulen und Lehrer haben es auch mit kritischen Adressaten zu tun - gerade weil Kinder zur Schule gehen müssen. So sind Schüler häufig damit beschäftigt, das Verhalten ihrer Lehrer zu beobachten und auf Fehler, Schwächen, etc. hin abzuscannen. Sie beobachten, ob der Lehrer sie beobachtet, oder ob sie sich vorübergehend außerhalb seines visuellen Kontrollbereichs befinden; sie versuchen, sich hinter dem Rücken ihrer Mitschüler zu verstecken; sie prätendieren, aufmerksam zuzuhören; sie mimen, als ob alles verstanden worden sei, etc. Wer als Schüler auf seine Individualität und Eigenständigkeit besteht, findet in dem Schulkontext und der Anwesenheit der Mitschüler ständig Anlass, nicht mitzumachen - und die Wahl dieser Alternative zu demonstrieren. 
auf. Die informative Distanz zwischen Elternhaus und Schule hat viele Konsequenzen (siehe Tyrell 1982). Aber sie erleichtert Schulkindern auch das Schwindeln und Vertuschen. So können Eltern es normalerweise nicht einschätzen, wie schwierig die bevorstehende Klassenarbeit oder Prüfung sein wird. Wer als Kind dem bevorstehenden Sonntagsspaziergang zu entkommen gedenkt, kann dieses Informationsdefizit der Eltern ausnutzen. Ähnlich sehen die Lehrer sich außerstande, die Schwierigkeiten in der Familie oder im Straßenverkehr zu beurteilen, auf welche die Schüler sich herausreden, wenn sie wieder einmal zu spät gekommen sind. Die Differenzierung dieser beiden Teilsysteme bringt es mit sich, dass Kinder auch in dieser Richtung sozialisiert werden.

e) Die Intransparenz und Nichtsteuerbarkeit des Schulgeschehens für die Elternhäuser hat noch eine weitere, meist übersehene Dimension, die nicht minder prekär ist oder doch sein kann. Es geht um die von den Eltern kaum und auch vom Lehrer nur in geringem Maße steuerbare Eigendynamik in den Beziehungen der Schüler untereinander, um die Gruppen- und Subkulturbildung (primär) unter den Klassenkameraden. Zwar beginnen Schulklassen meist an einem sozialen Nullpunkt: Die jeweils zu einer Klasse zusammengestellten Schüler kennen einander nicht oder nur zu einem geringen Teil. Gleichwohl setzen sofort fast zwangsläufig und fast immer auch mit großer Geschwindigkeit gruppendynamische Prozesse ein, welche unter anderem Beziehungspräferenzen sich einspielen lassen.

Klar ist: Weder der Lehrer noch die Eltern können solche Prozesse kontrollieren. Die Eltern haben es kaum in der Hand, mit wem ihr Sohn in der Klasse Umgang hat, welcher Clique er sich anschließt, an wen er sich feindselig oder freundschaftlich bindet, was er mit seinen Freunden in der Pause treibt etc. Immer können es in den Augen der Eltern gerade die falschen Freunde sein. Und dass das Klima in der Klasse sehr zum Leidwesen leistungsbejahender Eltern sich eher auf Lernunlust und die Prämierung und Prestigebesetzung von reichlich schulfernen Aktivitäten hin entwickeln kann, ist jedem Schulkenner geläufig. Was sich hier gruppendynamisch einspielt, ist in seiner Richtung meist nur schwer abänderbar und kaum reversibel; auch forcierte Lehrer-Eltern-Kooperation richtet gegen diese Eigendynamik meist nur wenig aus.

\section{IV.}

Es gibt im Bereich der Erziehungssoziologie eine wichtige Tradition, die sich bisher vor allem für die Schule interessiert hat. Seit Émile Durkheim (1903/1992: 127) wird hier die Schule beschrieben als ein sozialer „Mikrokosmos“, als ein Mikrokosmos der Gesellschaft in der Gesellschaft, dessen Funktion in der Transmission der dominanten Werte und Normen liegt. Dieser Ausgangspunkt hat dazu geführt, dass vor allem der Schule und der Korrespondenz zwischen Schule und Gesellschaft Aufmerksamkeit gewidmet wird. Die Anforderungen, die an das Verhalten in Unterrichtssituationen gestellt werden, werden als repräsentativ für die Anforderungen des täglichen Lebens in der modernen Gesellschaft gesehen - und dies nicht nur auf der Ebene des offiziellen Curriculums, sondern auch auf der Ebene der verborgenen oder latenten Strukturen einer leistungsorientierten Modernität. Man hat den Eindruck, dass die Erziehungssoziologie sich hier weiterhin auf eine unsichtbare Hand stützt, die die gesell- 
schaftliche Integration sicherstellen kann. Diese Ausgangspunkte haben aber gleichzeitig auch zur Folge, dass die Innendifferenzierung des Erziehungssystems weitgehend aus dem erziehungssoziologischen Blickfeld geraten ist.

Die hier präsentierten Analysen bieten keine ausgearbeitete Analyse des Verhältnisses von Familie und Schule. Wir haben das Verhältnis als Innendifferenzierung des Erziehungssystems und diese Innendifferenzierung vor dem Hintergrund der Ausdifferenzierung dieses Funktionssystems analysiert. Dabei ging es uns keineswegs um bloße Umetikettierung. Vielmehr ist damit gemeint, dass man das Verhältnis von Familie und Schule in den Kontext der Theorie der gesellschaftlichen Differenzierung hineinstellt und eine Fülle von Gesichtspunkten und Fragen gewinnt, die über den bisherigen Diskussionsstand hinausführen und die ihr Licht auch auf die erwähnten pädagogischen Schwierigkeiten mit der Sache werfen. Wir haben versucht, das Problemarsenal und die Denkmittel der Theorie der gesellschaftlichen Differenzierung daraufhin auszuloten, was sie für eine adäquate Konzeptualisierung des Verhältnisses von Familie und Schule hergeben. Unseres Erachtens können auf diese Art und Weise wichtige soziologische Fortschritte erreicht werden. Diese Fortschritte können sich als ein maßgeblicher Faktor erweisen, der es erlaubt, die theoretische Tradition des Fachs mit historischen und empirischen Daten zu verbinden.

\section{Literatur:}

Alexander, Jeffrey C./Colomy, Paul (Hg.) (1990): Differentiation Theory and Social Change: Comparative and Historical Perspectives. New York: Columbia University Press.

Ariès, Philippe (1960): L'enfant et la vie familiale sous l'Ancien Régime. Paris: Plon.

Baggerman, Arianne/Dekker, Rudolf (2005): Kind van de toekomst: De wondere wereld van Otto van Eck (1780-1798). Amsterdam: Wereldbibliotheek.

Bauman, Kurt J. (2001): Home Schooling in the United States: Trends and Characteristics. Washington: US Census Bureau (Population Division Working Paper Series Nr. 53).

Campe, Joachim Heinrich (Hg.) (1785): Allgemeine Revision des gesamten Schul- und Erziehungswesens: Von einer Gesellschaft praktischer Erzieher. Bd. 3. Hamburg: Ernst Bohn.

Claessens, Dieter (1967): Familie und Wertsystem: Eine Studie zur ,zweiten, sozio-kulturellen` Geburt des Menschen. Berlin: Duncker \& Humblot.

Curtis, Stanley J./Boultwood, Myrtle E. A. (1962): An Introductory History of English Education since 1800. London: University Tutorial Press.

Danziger, Kurt (1974): Sozialisation: Konzeptionelle Probleme, Methodologie und Ergebnisse. Düsseldorf: Schwann. de Condorcet, Jean Antoine Nicolas de Caritas (1847): Sur 1'instruction publique. In: Ders.: Euvres, Tome 7. Paris: Didot: 167-573.

de Perponcher, Willem E. (1774): Instructions d'un père à son fils. Utrecht: Spruit.

de Perponcher, Willem E. (1782): Onderwijs voor kinderen. 3 Bde. Utrecht: Schoonhoven.

Douthwaite, Julia V. (2002): The Wild Girl, Natural Man, and the Monster: Dangerous Experiments in the Age of Enlightenment. Chicago: University of Chicago Press.

Dreeben, Robert (1968): On What is Learned in School. Reading, Mass.: Addison-Wesley.

Durkheim, Émile (1992): L'education morale. Paris: Quadrige/Presses Universitaires de France.

Edgeworth, Richard L./Edgeworth, Maria (1801/1992): Practical Education. 3 Bde. London: Routledge.

Ehlers, Martin (1766): Gedanken von den zur Verbesserung der Schulen nothwendigen Erfordernissen. Altona/Lübeck: Iversen.

Fend, Helmut (1980): Theorie der Schule. München: Urban-Schwarzenberg.

Fend, Helmut (2006): Neue Theorie der Schule. Wiesbaden: VS Verlag für Sozialwissenschaften.

Gilgenmann, Klaus (1994): Die Familie als Erziehungsgemeinschaft: Zur Beschreibung der Funktion von Familien in der modernen Gesellschaft. In: System Familie 7: 212-228. 
Guthrie, Thomas (1973): Seed-time and Harvest of Ragged Schools. Montclair N. J.: Patterson Smith (zuerst 1860) [siehe auch Online-Quelle: www.newble.co.uk/guthrie/plea1.html].

Hans, Nicholas (1951): New Trends in Education in the Eighteenth Century. London: Routledge \& Kegan Paul.

Heywood, Colin (2001): A History of Childhood: Children and Childhood in the West from Medieval to Modern Times. Oxford: Blackwell.

Imelman, Jan Dirk (1998): Kinderopvang: Een discutabel antwoord op een maatschappelijk probleem. In: Levering et al. (1998): 142-147.

Inkeles, Alex/Sirowy, Larry (1983): Convergent and Divergent Trends in National Educational Systems. In: Social Forces 62: 303-333

Kay-Shuttleworth, James (1993): Four Periods of Public Education. London: Routledge (zuerst 1862).

la Vopa, Anthony J. (1988): Grace, Talent, and Merit: Poor Students, Clerical Careers, and Professional Ideology in Eighteenth-Century Germany. Cambridge: Cambridge University Press.

Levering, Bas/Biesta, Gert/Weijers, Ido (Hg.): Thema's uit de wijsgerige en historische pedagogiek. Utrecht: SWP.

Locke, John (1878): Gedachten over opvoeding (zuerst 1693). Groningen: Versluys.

Luhmann, Niklas/Schorr, Karl Eberhard (1982): Personale Identität und Möglichkeiten der Erziehung. In: Dies. (Hg.): Zwischen Technologie und Selbstreferenz: Fragen an die Pädagogik. Frankfurt am Main: Suhrkamp: 224-261.

Luhmann, Niklas/Schorr, Karl Eberhard (1988): Reflexionsprobleme im Erziehungssystem. Frankfurt am Main: Suhrkamp.

Maclure, Maggie/Walker, Barbara M. (2000): Disenchanted Evenings: The Social Organization of Talk in Parent-Teacher Consultations in UK Secondary Schools. British Journal of Sociology of Education 21: $5-25$.

Markowitz, Jürgen (1982): Relevanz im Unterricht - eine Modellskizze. In: Luhmann/Schorr (1982): 87-115.

Markowitz, Jürgen (1986): Verhalten im Systemkontext: Zum Begriff des sozialen Epigramms. Frankfurt am Main: Suhrkamp.

Matthes, Joachim (Hg.) (1980): Lebenswelt und soziale Probleme: Verhandlungen des 20. Deutschen Soziologentages zu Bremen 1980. Frankfurt am Main: Campus Verlag.

Millar, Hugh (1865): An Autobiography: My Schools and Schoolmasters; or, The Story of my Education. Boston: Gould \& Lincoln.

Mulcaster, Richard (1581): Positions Concerning the Training Up of Children. London: Vautrollier.

Musgrove, Frank (1966): The Family, Education and Society. London: Routledge \& Kegan Paul.

Niemeyer, August Hermann (1970): Grundsätze der Erziehung und des Unterrichts für Eltern, Hauslehrer und Erzieher. Herausgegeben von Hans-Hermann Groothoff u. Ulrich Herrmann. Paderborn: Schöningh (zuerst 1796).

Oelkers, Jürgen/Tenorth, Heinz-Elmar (Hg.) (1987): Pädagogik, Erziehungswissenschaft und Systemtheorie. Weinheim et al.: Beltz.

Parsons, Talcott (1955): The American Family: Its Relations to Personality and to the Social Structure. In: Ders./Bales, /Robert F. (Hg.): Family, Socialization and Interaction Process. London: Routledge \& Kegan Paul: 3-33.

Parsons, Talcott (1972): Das System moderner Gesellschaften. München: Juventa-Verlag.

Parsons, Talcott/Platt, Gerald M. (1973): The American University. Cambridge: Harvard University Press.

Priestley, Joseph (1995): Miscellaneous Observations Relating to Education (zuerst 1788). Bristol: Thoemmes.

Ramirez, Francisco O./Boli, John (1987): The Political Construction of Mass Schooling: European Origins and Worldwide Institutionalization. Sociology of Education 60: 2-17.

Reeve, Clara (1974): Plans of Education. New York: Garland (zuerst 1792).

Rousseau, Jean-Jacques (1991): Emile; or On Education. Harmondsworth: Penguin Books (zuerst 1762).

Smelser, Neil J. (1990): The Contest Between Family and Schooling in Nineteenth-Century Britain. In: Alexander/ Colomy (1990): 165-186.

Smelser, Neil J. (1991): Social Paralysis and Social Change: British Working-Class Education in the Nineteenth Century. Berkeley: University of California Press.

Spencer, Herbert (1904): An Autobiography. 2 Bde. London: Williams und Norgate.

Stichweh, Rudolf (1991): Der frühmoderne Staat und die europäische Universität: Zur Interaktion von Politik und Erziehungssystem im Prozess der Ausdifferenzierung (16.-18. Jahrhundert). Frankfurt am Main: Suhrkamp.

Trapp, Ernst C. (1977): Versuch einer Pädagogik: Von der Notwendigkeit, Erziehen und Unterrichten als eine eigene Kunst zu studieren. Nachdruck der 1. Ausgabe Halle 1780. Paderborn: Schöningh. 
Tyrell, Hartmann (1980): Soziologische Überlegungen zur Struktur des bürgerlichen Typus der Mutter-KindBeziehung. In: Matthes (1980): 417-428.

Tyrell, Hartmann (1982): Familienalltag und Familienumwelt. Überlegungen aus systemtheoretischer Perspektive. Zeitschrift für Sozialisationsforschung und Erziehungssoziologie 2: 167-188.

Tyrell, Hartmann (1987): Die ,Anpassung‘ der Familie an die Schule. In: Oelkers/Tenorth (1987): 102-124.

Vanderstraeten, Raf (2004): The Social Differentiation of the Educational System. In: Sociology 38: 255-272.

Vanderstraeten, Raf (2006): Soziale Beobachtungsraster: Eine wissenssoziologische Analyse von statistischen Klassifikationsschemata. In: Zeitschrift für Soziologie 35: 193-211.

Vatebender, Gerardus Carolus Conradus (1801): Antwoord op de Vraage: Welke wijze van Opvoeding is de meest verkiezelijke? In: Verhandelingen van het Provinciaal Utrechtsch Genootschap van Kunsten en Wetenschappen, IX. Utrecht: Wild \& Altheer.

Villaume, Peter (1785): Ob und in wie fern bei der Erziehung die Vollkommenheit des einzelnen Menschen seiner Brauchbarkeit aufzuopfern sey? In: Campe (1785): 435-616.

Wahrman, Dror (2004): The Making of the Modern Self: Identity and Culture in Eighteenth-Century England. New Haven: Yale University Press.

Weick, Karl E. (1979): The Social Psychology of Organizing, Second Edition. New York: Random House.

Wilderspin, Samuel (1840): The Infant System. London: Hodson [siehe auch Online-Quelle: www.fullbooks.com/ The-Infant-System1.html].

Williams, David (1774): A Treatise on Education. London: Payne.

Wolff, Betje (1977): Proeve over de opvoeding: Aan de Nederlandsche moeders. Meppel: Boom (zuerst 1780). 